\title{
Light diffusion and localization in 3D nonlinear disordered media
}

\author{
C. Conti $^{1,2}$, L. Angelani ${ }^{3}$, G. Ruocco ${ }^{2,4}$ \\ ${ }^{1}$ Centro studi e ricerche "Enrico Fermi," Via Panisperna 89/A, I-00184, Roma, Italy \\ ${ }^{2}$ Research center Soft INFM-CNR, c/o Università di Roma "La Sapienza," I-00185, Roma, Italy \\ ${ }^{3}$ Research center SMC INFM-CNR, c/o Università di Roma "La Sapienza," I-00185, Roma, Italy \\ ${ }^{4}$ Dipartimento di Fisica, Università di Roma "La Sapienza," I-00185, Roma, Italy
}

(Dated: April 9, 2018)

\begin{abstract}
Using a 3D Finite-Difference Time-Domain parallel code, we report on the linear and nonlinear propagation of light pulses in a disordered assembly of scatterers, whose spatial distribution is generated by a Molecular Dynamics code; refractive index dispersion is also taken into account. We calculate the static and dynamical diffusion constant of light, while considering a pulsed excitation. Our results are in quantitative agreement with reported experiments, also furnishing evidence of a non-exponential decay of the transmitted pulse in the linear regime and in the presence of localized modes. By using an high power excitation, we numerically demonstrate the "modulational instability random laser": at high peak input powers energy is transferred to localized states from the input pulse, via third-order nonlinearity and optical parametric amplification, and this process is signed by a power-dependent non-exponential time-decay of the transmitted pulse.
\end{abstract}

Electromagnetic wave localization and diffusion in random media have been recently considered by several authors. Experimental studies reported on various evidences of light localization, including the existence of a non-exponential long-time tail of the transmitted intensity [1, 2, 3, 4, 5, 6, 7, 8], and these processes were considered in numerous theoretical and numerical papers (as e.g. in 99, 10, 11, 12, 13, 14, 15, 16]). In a purely diffusive regime, pulsed light impinging on a disordered sample is rapidly dispersed while propagating; this implies that nonlinear effects are typically negligible. This holds true as far as localization processes do not get involved. For example, in random lasers (for a recent review see [17]), localized or extended modes [18] are excited in a disordered medium by means of an optically active material; in this case, the large resonant nonlinear susceptibility and the fact that long living (high Q) modes are excited foster a variety of nonlinear phenomena (as e.g. in [19, 20, 21]). For non-resonant ultra-fast nonlinearities (as $\chi^{(3)}$ processes of electronic origin [22]), it is in principle possible to use high-peak intensity femtosecond laser pulses to induce nonlinear effects, even in the presence of strong light diffusion. However, the (numerical and theoretical) analysis is enormously complicated by the need to include various effects as disorder, nonlinearity, material dispersion and to take into account a 3D environment. Even in the absence of nonlinearity, no "ab initio" numerical investigation of 3D Maxwell equations for femtosecond pulses in disordered media has been reported, to the best of our knowledge. Some theoretical investigations considered the role of the Kerr effect (i.e. an intensity dependent refractive index) for a monochromatic excitation [23, 24, 25, 26]; however ultra-fast $\chi^{(3)}$ optical nonlinearity may also provide other mechanisms. We specifically consider the gain of non-resonant origin, i.e. the optical parametric oscillation (OPO). OPO, in isotropic media in the absence of disorder, has been recently considered with reference to various devices [27, 28, 29], while in the fiber-optics community it is known as the "modulational instability laser" 30]. Here we report on the extensive $3 \mathrm{D}+1$ numerical analysis of linear diffusion and nonlinear amplification processes in a random dispersive nonlinear medium. We use a Molecular Dynamics (MD) code for generating a three dimensional distribution of spherical scatterers and a Finite-Difference Time-Domain (FDTD) parallel algorithm for simulating light propagation. The MD-FDTD approach not only reproduces the diffusive regime in agreement with reported experimental results in the linear regime, but also provides clear indications of the random OPO process, with analogies to random lasers.

An "ab initio" investigation of the nonlinear light propagation in 3D disordered media, requires a realistic distribution of a colloidal medium and of its nonlinear dielectric response. We model the disordered system as an assembly of poly-dispersed particles in air. The MD code generates the particle configuration; specifically, we considered a 50:50 mixture of spheres with diameter ratio 1.2 , interacting by a $100-200$ Lennard Jones potential 31]. The considered sample is composed by 1000 scatterers; once determined the particle positions by the MD code, each of them is taken as filled by a dispersive optically nonlinear medium, which is modeled as described below. The particle dimensions are chosen in order to simulate typical samples used in light localization and diffusion experiments, as e.g. in [1, 5]. We considered diameters for the biggest particles in the mixture ranging from $200 \mathrm{~nm}$ to $400 \mathrm{~nm}$; correspondingly the volume filling fraction $\phi$ for our samples, with volume $L^{3}(L=4$ $\mu \mathrm{m}$, of the order of the values considered in [6]), varies in the interval $\phi \in[0.1,0.6]$. The optical linear response of the particle medium is chosen as the best approximation of that of $\mathrm{TiO}_{2}$, used in most of the reported experiments, including material dispersion. The latter is 
modeled by a single-pole Lorentz medium, reproducing the data for $\mathrm{TiO}_{2}$ reported in [32]. Material absorption is also included providing an absorption coefficient $\kappa_{i}=10$ $\mathrm{mm}^{-1}$ at $\lambda=514 \mathrm{~nm}$. The 3D Maxwell equations, solved by the parallel FDTD [33] algorithm, are written as

$$
\begin{aligned}
& \nabla \times \mathbf{E}=-\mu_{0} \partial_{t} \mathbf{H} \quad \nabla \times \mathbf{H}=\epsilon_{0} \epsilon_{\infty} \partial_{t} \mathbf{E}+\partial_{t} \mathbf{P} \\
& \partial_{t}^{2} \mathbf{P}+2 \gamma_{0} \partial_{t} \mathbf{P}+\omega_{0}^{2} f(P) \mathbf{P}=\epsilon_{0}\left(\epsilon_{s}-\epsilon_{\infty}\right) \omega_{0}^{2} \mathbf{E}
\end{aligned}
$$

The non-resonant nonlinear response is determined by the nonlinear Lorentz oscillator (see e.g. [22]). As $f(P)=1$, eqs.(11) model the linear dispersive medium $\mathrm{TiO}_{2}$; by appropriately choosing $f(P)$, the system displays a isotropic ultra-fast nonlinearity, as previously described in [27], including the non-resonant $\chi^{(3)}$ susceptibility. In the present case, the associated Kerr effect has a nonlinear refractive index $\Delta n=n_{2} I$, with $I$ the optical intensity and $n_{2} \cong 10^{-18} \mathrm{~m}^{2} \mathrm{~W}^{-1}$. The parameters in $\mathrm{TiO}_{2}$ particles are given by (MKS units) : $\epsilon_{s}=5.9130$, $\epsilon_{\infty}=2.8731, \omega_{0}=6.6473 \times 10^{15}, \gamma_{0}=8.9168 \times 10^{11}$ and $f(P)=\left[1+\left(P / P_{0}\right)^{2}\right]^{3 / 2}$ with $P_{0}^{-2}=3.0$; out of the particles, $P=0$ and $\epsilon_{s}=\epsilon_{\infty}=1$ (vacuum).

The code is parallelized using the "MPI" protocol, and "UPML" absorbing boundary condition are used in the three spatial directions [33]. The used discretization is $\Delta x=\Delta y=\Delta z \cong 30 \mathrm{~nm}$ and $\Delta t \cong 0.02 \mathrm{fs}$. The $z$-propagating input beam is taken with a Gaussian $\mathrm{TEM}_{00}$ linearly $y$-polarized profile, at the input face of the disordered medium its waist is $w_{0}=1 \mu \mathrm{m}$. The input pulse temporal profile is Gaussian, with duration $t_{0}=100 \mathrm{fs}$ and carrier wavelength $\lambda=520 \mathrm{~nm}$. The numerical results have been verified a number of times by halving the spatio-temporal discretization. The validity of our approach has been confirmed in a variety of studies, as e.g. in [27, 34].

The first numerical simulations were aimed to investigate light diffusion when the nonlinear effects can be taken as negligible (low power), for a fixed sample spatial extension. We considered increasing values for the diameter of the spheres in the mixture; in the following $\sigma$ is the corresponding value of the biggest particles in the considered 50:50 mixture (for the others, the diameter is $\sigma / 1.2$ ). This corresponds to increase the average density of the medium, as well as the volume packing fraction. In order to unveil the onset of diffusion, we monitored the time-dependent output intensity, which is expected to develop an exponential tail in the diffusive regime. This is obtained in two ways: i) we considered the electric field $E_{y}$ at one point in the middle of the output face of the sample (see figure 1), and we calculated the "local intensity" by squaring the field and filtering with a low pass filter to remove the optical carrier (thus mimicking a photodiode); ii) we considered the total transmission from the output face of the sample as the calculated $z$-component of the Poynting vector, integrated with respect to the transverse $x, y$ coordinates.
In figure 1 we show the results for the local intensity, while increasing the volume filling fraction $\phi$. In order to compare the trailing edge of the transmitted pulses, for each signal we rescaled the peak value to the unity and shifted the temporal axis to make all the peaks for any $\sigma$ coincident. At high packing fraction the onset of an exponential trend is clearly visible. Note the residual oscillations, that are due to the fact that light is collected in a specific point at the output face. A typical far-field speckle pattern calculated from our simulation is shown in the inset of the bottom panel, and obtained by storing the $E_{y}$ component of the electric field in the output section, Fourier transforming with respect to the transverse coordinates, and averaging over time (the pattern is calculated for a continuous wave excitation at $\lambda=520$ $\mathrm{nm})$.

The intensity modulation in figure 1 disappears when considering the overall transmission from the output facet, which provides an average information over all the speckles and is shown in figure 2. The instantaneous flux for the z-component of the Poynting vector is calculated and then filtered to remove the reactive component. The appearance of an exponential tail in the transmission denotes the transition to the diffusive regime. The transmitted signal is $I(t) \propto \exp \left[-\pi^{2} D(t) t / L^{2}\right]$, with an instantaneous diffusion coefficient $D(t) \rightarrow D$ as $t \rightarrow \infty$ and a sample length $L$. When the packing fraction increases, the tail gets longer due to the reduced diffusivity $D$. The latter quantity is shown in Fig. 2(b) vs $\phi$. The results are in agreement with experimentally determined values, as reported in literature (e.g. in [1, 5, 6] ). Note that, as expected, $D$ decreases as the volume packing fraction increases, and at high $\phi$ the diffusivity tends to an asymptotic value (for non overlapping particles). The inset in Fig. 2(b) shows the calculated instantaneous diffusivity for two values of $\sigma$, which reproduces the reported trends, including some temporal oscillations [1, 4 ].

Next, we consider the diffusive regime for a fixed $\sigma=400 \mathrm{~nm}(\phi \cong 0.6)$, while increasing the input peak power $P_{i n}$. In figure 3(a), we show the local intensity for two input peak powers; the onset of a non-exponential tail is evident, as also shown in the inset reporting the intensity auto-correlation function. In the panel (b) we show the transmitted intensity (integrated Poynting vector) for various input powers. The inset shows the instantaneous diffusivity that drops at high powers and long times, when a threshold value for the input peak power is reached. These results foster the interpretation of the data in terms of a light-induced localization transition, strongly resembling that controlled e.g. by the packing fraction and experimentally observed (as e.g. in 1]). However, in the present case, the transition is controlled by the input power, and hence it has a nonlinear origin. It will be shown below that it can be explained as the excitation of a "modulational instability random laser" (or random OPO). 
Indeed, to unveil the underlying mechanism, we resorted to the spectral analysis of the electric field in various points inside the random medium; figure 4 summarizes the typical result. In panel (a) we show the calculated frequency spectra of the electric field $E_{y}$ in the random structure, for different input peak powers. The data are displayed in logarithmic scale and vertically shifted by an arbitrary amount for the sake of clearness. At low power the spectrum of the input pulse is reproduced, with the addition of small ripples (due to the vertical scale in figure 4 a) in correspondence of the existing high$\mathrm{Q}$ modes. As the power increases, the bandwidth gets larger and regions with depression and enhancement of the spectrum appear. At sufficiently high power, a wide band excitation is found. This behavior can be explained by recalling that in $\chi^{(3)}$ media energy can be transferred from various harmonics to others through parametric amplification. We stress that the nonlinear effects are weakly pertubative, indeed we consider power level just above the OPO threshold and the nonlinear index perturbation, due to the Kerr effect, is of the order of 0.001. Thus the localization process in figure 3 is explained by assuming that energy is nonlinearly transferred to localized, long-living states.

In panel (b) we show the time evolution of the spectrum (i.e. the spectrogram calculated by Fourier transforming windowed parts of the temporal signal) at $P_{\text {in }}=$ $3 \mathrm{~kW}$. In the initial interval $t<1 \mathrm{ps}$, energy is transferred to a wide band around the input pulse spectrum. However, at large times only the long-lifetime (high Qfactor) modes survive and the spectrum is characterized by several peaks in the region around $\lambda \cong 300 \mathrm{~nm}$ $\left(\nu \cong 0.9 \times 10^{15} \mathrm{~Hz}\right)$. In order to sustain the fact that localized modes put into oscillation, we show in the inset of figure $4(\mathrm{c})$ a snapshot of the electric field $E_{y}$ in the sample, taken at $P=2.5 \mathrm{~kW}$ and $t \cong 4$ ps which clearly show the occurrence of localized excitations.

In addition, we went back to the linear regime to analyze the localized modes are supported in our virtual samples. First, we repeated the pulse transmission experiment at low power $P=1 \mathrm{nW}$ and central wavelength $\lambda=300 \mathrm{~nm}(\sigma=400 \mathrm{~nm})$ and Figure 4(c) clearly shows an non-exponential tail at $t \cong 2.5 \mathrm{ps}$, which is not present for $\lambda=521 \mathrm{~nm}$ (figure 2a) also for $t>3 \mathrm{ps}$ (not shown). Then, we recall that light localization is typically expected in proximity of depressions of the density of states (DOS). For the case of weakly disordered periodical structures, these are obtained inside the photonic band gaps [35], while, for random dispersions of particles, the DOS (which is numerically calculated by shining the sample by a ultra-wide band single-cycle pulse and spectrally analyzing the transmitted pulse) displays some depressions (pseudo-gaps) in proximity of the Mie resonances (see e.g. [3, 11]). In our case, the distribution of states displays various depressions with the most pronounced around $0.9 \times 10^{15} \mathrm{~Hz}$, as shown in Fig. 4(d).
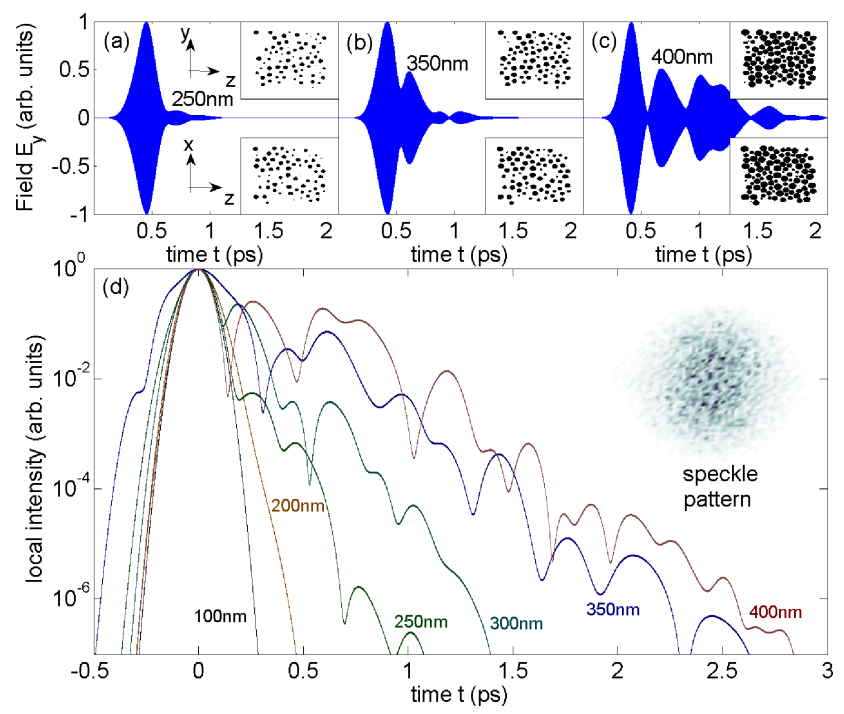

FIG. 1: (Color online) Top panels (a-c): electric field in the middle of the output face of the sample at low input peak power $\left(P_{\text {in }}=1 \mathrm{nW}\right)$, for three different particle diameters $(\sigma=250,350,400 \mathrm{~nm})$. The insets show the particle distribution in the yz (top) and $\mathrm{xz}$ (bottom) middle section of the sample. Bottom panel (d): local intensity in logarithmic scale. The inset shows the calculated speckle pattern.
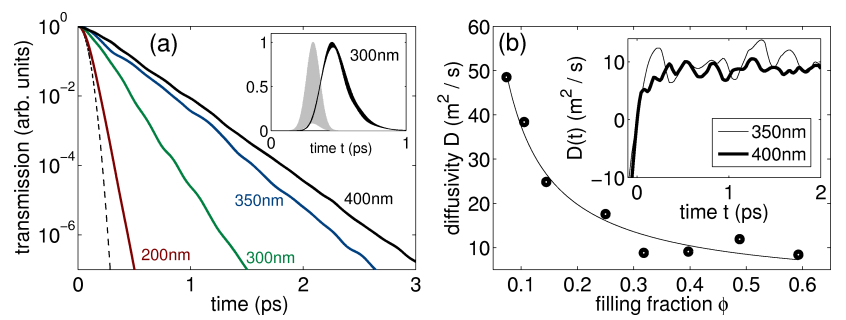

FIG. 2: (Color online) (a) Tail of the transmitted pulse (the flux of the z-component of the Poynting vector at the output face) for various particle diameters. The inset shows the transmitted pulse in linear scale, the gray region is the input pulse. (b) Calculated diffusion constant for various filling fractions. The continuous line is a best-fit by an inverse power law. The inset shows the dynamical diffusivity for two values of the particle diameters.

The fact that localized modes do exist in proximity of the pseudo-gaps, is also confirmed by the plot of the calculated Q-factor vs frequency (bold line in Fig. 4(d)), realized by using an harmonic inversion library [36]. Which of the localized modes are effectively excited will depend not only on the Q-factors, but also on the spatial overlaps with the pump modes and with the spatial distribution of the nonlinear susceptibility (the $g$ coefficient discussed below). The localized modes are a reminiscence of the Mie resonances of the isolated spheres, which are strongly affected by the presence of adjacent resonators in a disordered configuration, in analogy to what happen for periodical mono-dispersed photonic crystal, investigated e.g. 


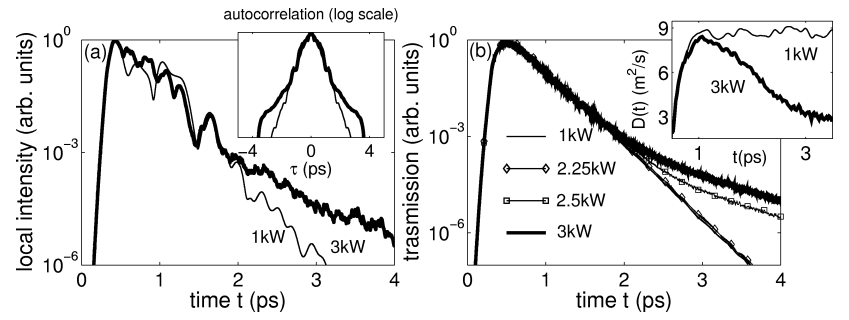

FIG. 3: (a) Time dynamics of the local intensity for two different input peak powers; the inset shows the corresponding auto-correlation functions versus the delay $\tau$; (b) transmitted signal for various input peak powers; the inset shows the calculated instantaneous diffusivity.
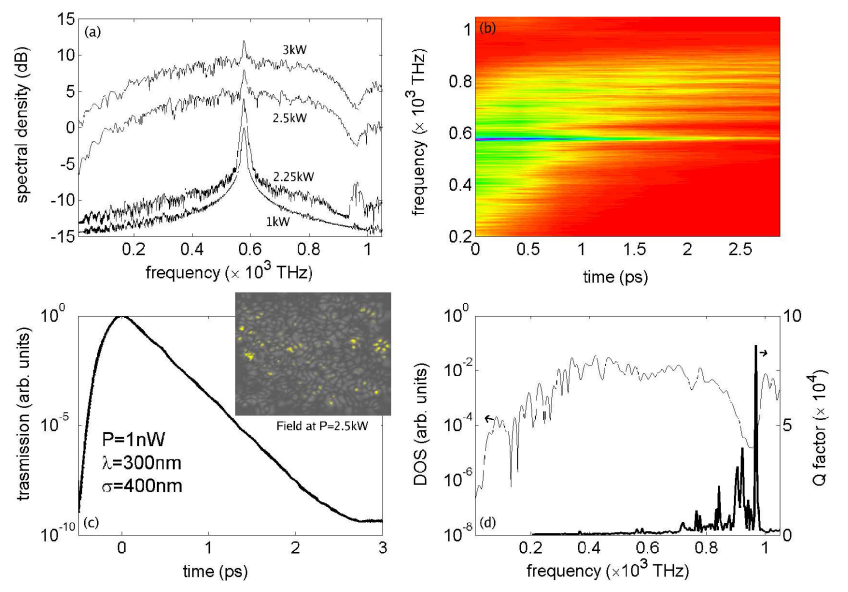

FIG. 4: (Color online) (a) Spectral emission for increasing peak power; (b) spectrogram displaying the time evolution of the spectra for $P=3 \mathrm{~kW}$; (c) transmission of a low power pulse at $\lambda=300 \mathrm{~nm}$ and $\sigma=400 \mathrm{~nm}$ displaying a notexponential tails; the inset shows the field distribution for $\sigma=400 \mathrm{~nm}$ at high power $(P=2.5 \mathrm{~kW})$ at $t=4 \mathrm{ps}$ in the xz middle section of the sample; (d) density of states and Q-factor distribution for $\sigma=400 \mathrm{~nm}$.

in 37.

Before concluding we show that the basic features of the reported results can be reproduced by a simple theoretical model, based on the coupled mode theory in the time domain (CMT) [38]. Indeed the small-signal parametric gain for the four-waves interaction $\omega_{0}+\omega_{0}=$ $\left(\omega_{0}+\Omega\right)+\left(\omega_{0}-\Omega\right)$, can be determined by introducing the complex amplitudes for the pump mode $a_{0}(t)=a\left(\omega_{0}, t\right)$ and for the generated modes $a_{ \pm}(t)=a\left(\omega_{0} \pm \Omega, t\right)$. The complex amplitudes are normalized in a way such that $|a(\omega, t)|^{2}$ is the energy stored in the mode at angular frequency $\omega$, and we denote with $\tau(\omega)$ the lifetime, and $Q(\omega)=\omega \tau(\omega) / 2$ the corresponding Q-factor. In the undepleted pump approximation the relevant CMT equations are written in compact and obvious notation as

$$
\frac{d a_{ \pm}}{d t}=-\frac{1}{\tau_{ \pm}} a_{ \pm}+i g a_{0}^{2} a_{\mp}^{*} .
$$

The coefficient $g$ is the relevant 3D spatial overlap be- tween the mode electric field distributions and the nonlinear third-order susceptibility. Looking for solutions in the form $a_{ \pm}=A_{ \pm} \exp (\alpha t)$, the real-valued gain $\alpha$ is given by

$$
\alpha=-\left(\frac{1}{2 \tau_{+}}+\frac{1}{2 \tau_{-}}\right)+\left[\left(\frac{1}{2 \tau_{+}}-\frac{1}{2 \tau_{-}}\right)^{2}+\left|g a_{0}^{2}\right|^{2}\right]^{1 / 2} .
$$

It is readily seen that a condition for having amplification $(\alpha>0)$ is given by $\left|g a_{0}^{2}\right|^{2}>1 /\left(\tau_{+} \tau_{-}\right)=\omega_{+} \omega_{+} / 4 Q_{+} Q_{-}$. Assuming that for all the involved modes $\omega \cong \omega_{0}$ and the Q-factor is almost the same, with the exception of those localized that display higher values, it is concluded that the localized modes (those with not negligible overlap $g$ with the pump modes) are put into oscillations at lower pump energies (see line at $2.25 \mathrm{~kW}$ in figure $4(\mathrm{a})$ ). Additionally the asymmetry, at low power, in the spectrum (see 4a) can be explained by observing from Eq. (2) that $\left|A_{+} / A_{-}\right|>1$ if $Q_{+}>Q_{-}$. At higher powers most of the modes are put into oscillation and the spectrum mimics the DOS.

In conclusion, the considered disordered, nonlinear and dispersive samples support localized modes in proximity of Mie resonances. These can be excited by highintensity pulses through optical parametric amplification. The overall process can be interpreted as the excitation of a "modulational instability random laser". The main difference with standard random lasers is given by the fact that an ultra-fast optical process provides the required gain and does not need doping of the sample by an active media. These results can be generalized to other nonlinear amplification processes, like the Raman effect, and can find application in all-optical storage of light, ultra-fast laser-tissue interaction, soft-matter and granular systems. We thank S. Trillo and D. Wiersma for fruitful discussions. We acknowledge support from the INFM-CINECA initiative for parallel computing.

[1] M. Storzer, P. Gross, C. M. Aegerter, and G. Maret, Phys. Rev. Lett. 96, 063904 (2006).

[2] A. A. Chabanov, B. Hu, and A. Z. Genack, Phys. Rev. Lett. 93, 123901 (2004).

[3] A. A. Chabanov and A. Z. Genack, Phys. Rev. Lett. 87, 153901 (2001).

[4] A. A. Chabanov, Z. Q. Zhang, and A. Z. Genack, Phys. Rev. Lett. 90, 203903 (2003).

[5] I. M. Vellekoop, P. Lodahl, and A. Lagendijk, Phys. Rev. E 71, 056604 (2005).

[6] P. M. Johnson, A. Imhof, B. P. J. Bret, J. G. Rivas, and A. Lagendijk, Phys. Rev. E 68, 016604 (2003).

[7] D. S. Wiersma, P. Bartolini, A. Lagendijk, and R. Righini, Nature 390, 671 (1997).

[8] F. Reil and J. E. Thomas, Phys. Rev. Lett. 95, 143903 (2005). 
[9] M. C. W. van Rossum and T. M. Nieuwenhuizen, Rev. Mod. Phys. 71, 313 (1999).

[10] S. E. Skipetrov and B. A. van Tiggelen, Phys. Rev. Lett. 96, 043902 (2006).

[11] C. Vanneste and P. Sebbah, Phys. Rev. E 71, 026612 (2005).

[12] S. K. Cheung and Z. Q. Zhang, Phys. Rev. B 72, 235102 (2005).

[13] P. Markos and C. M. Soukoulis, Phys. Rev. B 71, 054201 (2005).

[14] S. H. Tseng, J. H. Greene, A. Taflove, D. Maitland, V. Backman, V., and J. T. Walsh Jr., Opt. Lett. 29, 1393 (2004).

[15] S. H. Tseng, Y. L. Kim, A. Taflove, D. Maitland, V. Backman, and J. T. Walsh Jr., Opt. Express 13, 3666 (2005).

[16] S. H. Tseng, A. Taflove, D. Maitland, V. Backman, and J. T. Walsh Jr., Opt. Express 13, 6127 (2005).

[17] H. Cao, J. Phys. A. : Math. Gen. 38, 10497 (2005).

[18] S. Mujumdar, M. Ricci, R. Torre, and D. S. Wiersma, Phys. Rev. Lett. 93, 053903 (2004).

[19] B. Liu, A. Yamilov, Y. Ling, J. Y. Xu, and H. Cao, Phys. Rev. Lett. 91, 063903 (2003).

[20] L. I. Deych, Phys. Rev. Lett. 95, 043902 (2005).

[21] L. Angelani, C. Conti, G. Ruocco, and F. Zamponi, Phys. Rev. Lett. 96, 065702 (2006).

[22] R. W. Boyd, Nonlinear Optics (Academic Press, New York, 2002), 2nd ed.

[23] B. Spivak and A. Zyuzin, Phys. Rev. Lett. 84, 1970 (2000).
[24] S. E. Skipetrov and R. Maynard, Phys. Rev. Lett. 85, 736 (2000).

[25] S. E. Skipetrov, Phys. Rev. E 63, 056614 (2001).

[26] S. E. Skipetrov, Phys. Rev. E 67, 016601 (2003).

[27] C. Conti, A. Di Falco, and G. Assanto, Opt. Express 12, 823 (2004), arXiv:physics/0403013.

[28] M. A. Foster, A. C. Turner, J. E. Sharping, B. S. Schmidt, M. Lipson, and A. L. Gaeta, Nature 441, 960 (2006).

[29] T. J. Kippenberg, S. M. Spillane, and K. J. Vahala, Phys. Rev. Lett. 93, 083904 (2004).

[30] M. Nakazawa, K. Suzuki, and H. A. Haus, IEEE J. Quantum Electron. 25, 2036 (1989).

[31] L. Angelani, G. Foffi, F. Sciortino, and P. Tartaglia, J. Phys. : Condens. Matter 17, L113 (2005), we used the data at temperature $\mathrm{T}=0.26$ in the units of this reference.

[32] J. R. DeVore, J. Opt. Soc. Am. 41, 416 (1951).

[33] A. Taflove and S. C. Hagness, Computational Electrodynamics: the finite-difference time-domain method (Artech House, 2000), 3rd ed.

[34] C. Conti, A. Di Falco, and G. Assanto, Opt. Lett. 29, 2902 (2004).

[35] S. John, Phys. Rev. Lett. 58, 2486 (1987).

[36] V. A. Mandelshtam, Progress in NMR Spectroscopy 38, 159 (2001), we used the Harminv library by Steven G. Johnson (MIT).

[37] C. Vandenbem and J. P. Vigneron, J. Opt. Soc. Am. A 22, 1042 (2005).

[38] H. A. Haus, Waves and Fields in Optoelectronics (Prentice-Hall, Englewood Cliffs, N. J., 1984). 